\title{
Response of Sesame (Sesamum indicum L.) to Growth Regulator under Dry Farming Condition
}

\author{
G.B. Vekaria, K.D. Rakholiya, V.D. Vora*, J.T. Patel, G.S. Sutaria and P.D. Vekaria \\ Main Dry Farming Research Station, Junagadh Agricultural University, Targhadia, \\ Rajkot - 360003, Gujarat, India \\ *Corresponding author
}

\begin{tabular}{|c|c|}
\hline & A B S T R A C T \\
\hline $\begin{array}{l}\text { Crop growth } \\
\text { indexes growth } \\
\text { regulators, Capsule } \\
\text { formation, Sesame } \\
\text { (Sesamum indicum } \\
\text { L.), Yield. }\end{array}$ & \multirow{3}{*}{$\begin{array}{l}\text { A field experiment was conducted during the kharif season of } 2011 \text { and } 2014 \text { to study the } \\
\text { response of sesame (Sesamum indicum } \mathrm{L} \text {.) to plant growth regulators. The experiment was } \\
\text { conducted in Randomized Block Design with ten treatments and replicated thrice. Among } \\
\text { the different treatments } \mathrm{T}_{5}(\mathrm{IAA} @ 100 \mathrm{ppm} \text { at flowering has recorded maximum value for } \\
\text { plan height }(131.8 \mathrm{~cm}) \text { and maximum number of capsules per plant at } 70 \text { DAS }(55.5) \text {. The } \\
\text { significantly highest } 1000 \text { seed weight }(3.38 \mathrm{~g} \text { ) of sesame was recorded due to spraying of } \\
\text { IAA @ } 100 \mathrm{ppm} \text { at flowering }+ \text { capsules formation stage }\left(\mathrm{T}_{7}\right) \text { followed by } \mathrm{T}_{5} \text { (IAA @ } 100 \\
\text { ppm at flowering stage. The significantly highest harvest index }(25.8) \text { was noted under T3 } \\
\text { Gibberellic Acid }\left(\mathrm{GA}_{3}-100 \mathrm{ppm}\right) \text { at capsules formation stage. On the bases of pooled } \\
\text { results, the significantly highest seed }\left(947 \mathrm{~kg} \mathrm{~h}^{-1}\right) \text {, stalk }\left(2810 \mathrm{~kg} \mathrm{ha}^{-1}\right) \text { and biological } \\
\text { yield }\left(3757 \mathrm{~kg} \mathrm{ha}^{-1}\right) \text { were obtained due to foliar spraying of IAA @ } 100 \mathrm{ppm} \text { at flowering } \\
\text { and capsule formation stage }\left(\mathrm{T}_{7}\right) \text { and statically at par with } \mathrm{T}_{5} \text { and } \mathrm{T}_{6} \text {. }\end{array}$} \\
\hline Article Info & \\
\hline $\begin{array}{l}\text { Accepted: } \\
\text { 18 February } 2017 \\
\text { Available Online: } \\
\text { 10 March } 2017\end{array}$ & \\
\hline
\end{tabular}

\section{Introduction}

Sesame (Sesamum indicum L.) is one of the oldest oil seed crop cultivated in India. It is called as "Queen of oil seed crop" by virtue of its excellent quality. Sesame is very droughttolerant crop of semiarid regions. It is superior to other oilseed crop due to adaptability to varied agro-climatic condition and high degree of drought tolerance. It is widely grown in countries such as India, China, Bangladesh, Turkey and also in drier parts of African and Mediterranean countries.

It has been called a survivor crop, with an ability to grow where most crops fail. It is popularly known as gingelly, til, benni, ajanjoli, ellu, goma and simsim in different languages. Sesame ranks first for having oil content of 46-64 per cent and $6355 \mathrm{~K} \mathrm{cal} \mathrm{kg}^{-1}$ dietary energy in seeds (Sanjay Kumar and Goel, 1994). Seed of sesame is also rich source of protein (20-28\%), sugar (14-16\%) and minerals $(5-7 \%)$. This oil has 85 percent unsaturated fatty acid is highly stable and has washing effect on cholesterol and prevents coronary heart disease. Sesame as a valued oil seed appears to have numerous industrial applications.

Sesame originated from South West Africa and is botanically termed as Sesamum indicum L. of family Pedaliaceae. Among top ten oil seed producing countries Mayammar 
ranks first in production of 0.72 million tonnes with productivity of 0.46 tonnes $\mathrm{ha}^{-1}$ fallowed by India which having production of 0.62 metric tonnes and productivity of 0.34 tonnes ha ${ }^{-1}$. It is clearly indicated that, it is not matching with the increasing demand. The country continues to experience edible oil deficit (Hedge, 2002). A further increase in production could be achieved by adopting improved agronomic practices like proper nutrient management, application of biofertilizers, micronutrients and growth regulators, reorienting of plant structure, reducing field harvesting losses and storage losses. To achieve this strategy, the field experiment was conducted at Main Dry Farming Research Station, Junagadh Agricultural University, Targhadia with the entitled Response of sesame (Sesamum indicum L.) to growth regulator during monsoon period.

\section{Materials and Methods}

\section{Experimental site and plant material}

Field trials was carried out during the Kharif seasons 2011 to 2014 at main dry farming research station, Junaghadh Agriculture University, Targhadia, located at 22.17' N, $70^{\circ} 48^{\prime} \mathrm{E}$ and altitude $137.7 \mathrm{~m}$ above mean sea level. The research farm is situated in north Saurashtra agroclimatic zone of Gujarat. Soil of the experimental site was medium black with $\mathrm{pH} 8.1$ and organic carbon status $0.59 \%$. The area was ploughed and harrowed before sowing. Recommended dose of fertilizer was applied before sowing to each plot at 50.0:25.0:0.0 NPK.

The seeds of sesame (Sesamum indicum L.) variety "Gujarat Til-3"were sown in eight rows per plot, $5 \mathrm{~m}$ length with spacing of 45 $\mathrm{cm}$ between rows. After germination, the plants were thinned to obtain optimum plant population. Two inter-culturing and hand weeding were carried out. The three types of growth regulators of different concentration were foliar sprayed at flowering and capsules formation stage as following treatments.

\section{Treatments}

The experiment comprised of total 10 treatments viz. $\mathrm{T}_{1}\left[(\right.$ Absolute control $), \mathrm{T}_{2}$ (gibberellic acid (GA 3 -100ppm) at flowering stage)], $\mathrm{T}_{3}$ [(gibberellic acid $\left(\mathrm{GA}_{3}\right.$ $100 \mathrm{ppm})$ at capsules formation stage)], $\mathrm{T}_{4}\left[\right.$ (gibberellic acid $\left(\mathrm{GA}_{3}-100 \mathrm{ppm}\right)$ at flowering stage and capsules formation)], $\mathrm{T}_{5}$ [(Indole Acetic Acid (IAA -100ppm) at flowering stage $)], \mathrm{T}_{6}[(\mathrm{~T} 6$ Indole Acetic Acid (IAA -100ppm) at capsules formation stage)], $\mathrm{T}_{7}$ [(Indole Acetic Acid (IAA -100ppm) at flowering stage and capsules formation stage)], $\mathrm{T}_{8}$ [(Benzine Amino purine (BA -5 ppm) at flowering stage)], $\mathrm{T}_{9}$ [Benzine Amino purine (BA- $5 \mathrm{ppm}$ ) at capsules formation stage)] and $\mathrm{T}_{10}$ [Benzine Amino purine (BA$5 \mathrm{ppm}$ ) at flowering and capsules formation stage].

\section{Experimental design and layout}

The experiment was laid out thrice in Randomized Block Design (R.B.D.) in with the plot size of (a) Gross: $5.0 \mathrm{~m} \mathrm{x} 3.6 \mathrm{~m}$ (8 lines) (b) Net: $4.0 \mathrm{~m}$ x $2.7 \mathrm{~m}$ (6 lines). The row spacing and seed rate were $45 \mathrm{~cm} \times 10$ $\mathrm{cm}$ and $2.25 \mathrm{Kg} \backslash$ ha respectively. The fertilizer 50.0-25.0-0.0 NPK kg ha ${ }^{-1}$ was applied.

\section{Observations}

The plants were harvested at physiological maturity and yield components, such as plant height $(\mathrm{cm})$, number of total capsules per plant at 70 DAS and maturity, 1000 seed weight and harvest index were recorded on five randomly selected plants in each plot. Seed yield was determined by harvesting the middle six rows of each plot. 


\section{Harvest index}

Harvest index is defined as the yield of grain divided by the total yield of above ground biomass (Stover plus grain).

Harvest index $=$ yield of grain $/$ (yield of Stover + yield grain) $\times 100$

\section{Seed yield $\left(\mathrm{kg} \mathrm{ha}^{-1}\right)$}

At the maturity, the sesame crop in each plot was harvested and threshed, and yield $\mathrm{ha}^{-1}$ was calculated by the following formula

Seed yield plot $\mathrm{kg}^{-1}$ Seed yield $\mathrm{kg} \mathrm{ha}^{-1}=($ Seed yield plot $\mathrm{kg}^{-1} /$ Plot size $\mathrm{m}^{-2}$ ) X 10000

\section{Statistical analysis}

Statistical analysis of variance was done using SAS computer software package and the mean values were compared with Duncan multiple range test (DMRT) at 0.05 probability level.

\section{Results and Discussion}

\section{Effect of growth regulators on growth parameters}

Effect of growth regulators on growth parameters of sesame are presented in Table 1. In the results revealed that the growth parameters of sesame were significantly differed during pooled result due to foliar spraying of growth regulator. The highest value of plant height $(131.8 \mathrm{~cm})$ was observed with T5 (IAA @ 100 ppm at flowering stage) (Table 1a) which was at par with treatments $\mathrm{T}_{3}, \mathrm{~T}_{4}$ and $\mathrm{T}_{6}$ to $\mathrm{T}_{10}$. Pooled results indicated that the highest number of branches per plant (2.81) of sesame was recorded under spraying of IAA@ 100 ppm at flowering stage + capsules formation stage(T7) followed by T5 (IAA @ 100 ppm at flowering stage) (Table 1b).The minimum plant height $(123.7 \mathrm{~cm})$ and minimum number of branches (1.89) were observed under control plot.IAA induced higher plant height was reported earlier in grasspea (Rahman et al., 1989), soybean (Reena et al., 1999), groundnut (Lee, 1990), Mungbean (Quaderi et al., 2006), Jatropha curcas (Joshi et al., 2011) and tomato (Khaled et al., 2015). The stimulatory effects of IAA on plant height in the present experiment agreed well with the above findings.

Effect of foliar spraying of growth regulator on number of capsules plant ${ }^{-1}$ at 70 DAS and maturity as well as capsule growth rate was found significant. In pooled result significantly highest number of capsules per plant at 70 DAS (55.5) was observed with $\mathrm{T}_{5}$ (IAA @ 100 ppm at flowering stage) (Table 2a)which was at par with treatments $T_{3}, T_{4}$, $\mathrm{T}_{6}, \mathrm{~T}_{7}, \mathrm{~T}_{9}$ and $\mathrm{T}_{10}$. While at maturity treatment $\mathrm{T}_{7}$ (IAA @ 100 ppm at flowering + capsules formation stage Table 2b) recorded significantly highest number of capsules per plant (65.4) followed by $\mathrm{T}_{5}$ (IAA @ 100 ppm at flowering stage), $\mathrm{T}_{6}$ (T6 IAA $-100 \mathrm{ppm}$ at capsules formation stage) and $\mathrm{T}_{10}$ (BA- $5 \mathrm{ppm}$ at flowering and capsules formation stage). The minimum number of capsule per plant at 70 DAS and maturity stage were found under control condition. In sesame crop, spraying of IAA@ 100 ppm at flowering stage (T5) and GA3 @ 100 ppm at capsules formation stage (T3) was recorded significantly higher capsules growth rate. Senthil et al., (2003) also investigated the IAA at $100 \mathrm{ppm}$ supplied as foliar spray at 35 and 60 days after sowing on some biochemical and physiological aspects of soybean plant. They reported that all treatments increased the biochemical parameters of soybean and IAA treatment had the highest effects on the plant. Khaled et al., (2015) also reported that IAA treated plots showed better performance for growth parameters and yield compared to control condition and 100 ppm IAA was more suitable than the $200 \mathrm{ppm}$ IAA for higher yield for tomato cultivation. 
Table.1 Effect of growth regulators on growth parameters of sesame

\begin{tabular}{|c|c|c|c|c|c|c|c|}
\hline Sr. No. & Treatment & 2011 & 2013 & 2014 & Pooled & $\mathbf{Y}$ & $\mathbf{Y} \mathbf{x} \mathbf{T}$ \\
\hline \multicolumn{8}{|c|}{ 1(a) Plant Height (cm) } \\
\hline $\mathrm{T}_{1}$ & Control & 104.7 & 130.1 & 136.4 & 123.7 & & \\
\hline $\mathrm{T}_{2}$ & GA3 @ 100ppm at flowering stage & 103.2 & 131.9 & 137.5 & 124.2 & & \\
\hline $\mathrm{T}_{3}$ & GA3 @ 100ppm at capsule formation stage & 105.9 & 133.8 & 139.0 & 126.3 & & \\
\hline $\mathrm{T}_{4}$ & GA3 @ 100ppm at flowering stage + capsule formation stage & 97.7 & 135.1 & 142.7 & 125.1 & & \\
\hline $\mathrm{T}_{5}$ & IAA@100ppm at flowering stage & 104.5 & 142.3 & 148.5 & 131.8 & & \\
\hline $\mathrm{T}_{6}$ & IAA @ 100ppm at capsule formation stage & 105.7 & 141.4 & 147.2 & 131.4 & & \\
\hline $\mathrm{T}_{7}$ & IAA @ 100ppm at flowering stage + capsule formation stage & 98.1 & 144.1 & 149.5 & 130.6 & & \\
\hline $\mathrm{T}_{8}$ & BA @ 5ppm at flowering stage & 106.3 & 134.3 & 141.7 & 127.4 & & \\
\hline $\mathrm{T}_{9}$ & BA@ @ppm capsule Formation Stage & 110.6 & 135.8 & 142.6 & 129.7 & & \\
\hline \multirow[t]{4}{*}{$\mathrm{T}_{10}$} & BA @5ppm at flowering stage + capsule formation stage & 103.5 & 138.7 & 143.0 & 128.4 & & \\
\hline & S.Em. \pm & 3.37 & 3.64 & 3.87 & 2.24 & 1.23 & 3.63 \\
\hline & C.D.at $5 \%$ & 10.01 & 10.81 & 11.49 & 6.67 & 3.65 & 10.30 \\
\hline & C.V. $\%$ & 5.61 & 4.61 & 4.69 & 4.92 & & \\
\hline \multicolumn{8}{|c|}{ 1(b) Number of Branches/plant } \\
\hline $\mathrm{T}_{1}$ & Control & 1.67 & 2.21 & 1.80 & 1.89 & & \\
\hline $\mathrm{T}_{2}$ & GA3@100ppm at flowering stage & 1.93 & 2.47 & 2.00 & 2.13 & & \\
\hline $\mathrm{T}_{3}$ & GA3 @ 100ppm at capsule formation stage & 2.00 & 2.53 & 2.00 & 2.18 & & \\
\hline $\mathrm{T}_{4}$ & GA3 @ 100ppm at flowering stage + capsule formation stage & 2.13 & 2.67 & 2.10 & 2.30 & & \\
\hline $\mathrm{T}_{5}$ & IAA @ 100ppm at flowering stage & 2.33 & 3.11 & 2.57 & 2.67 & & \\
\hline $\mathrm{T}_{6}$ & IAA @ 100ppm at capsule formation stage & 2.27 & 2.99 & 2.33 & 2.53 & & \\
\hline $\mathrm{T}_{7}$ & IAA @ 100ppm at flowering stage + capsule formation stage & 2.53 & 3.23 & 2.67 & 2.81 & & \\
\hline $\mathrm{T}_{8}$ & BA@5ppm at flowering stage & 2.10 & 2.43 & 2.00 & 2.18 & & \\
\hline $\mathrm{T}_{9}$ & BA@ @ppm capsule Formation Stage & 2.20 & 2.90 & 2.10 & 2.40 & & \\
\hline \multirow[t]{4}{*}{$\mathrm{T}_{10}$} & BA @ $5 p p m$ at flowering stage + capsule formation stage & 2.20 & 2.80 & 2.00 & 2.33 & & \\
\hline & S.Em. \pm & 0.15 & 0.20 & 0.17 & 0.05 & 0.03 & 0.17 \\
\hline & C.D.at $5 \%$ & 0.44 & 0.60 & 0.49 & 0.16 & 0.09 & 0.49 \\
\hline & C.V. $\%$ & 12.07 & 12.77 & 13.31 & 12.82 & & \\
\hline
\end{tabular}

*Foliar spray at flowering and capsule formation stage. 
Table.2 Effect of growth regulators on growth parameters of sesame

\begin{tabular}{|c|c|c|c|c|c|c|c|}
\hline Sr. No. & Treatment & 2011 & 2013 & 2014 & Pooled & $\mathbf{Y}$ & $\mathbf{Y} \times \mathbf{T}$ \\
\hline \multicolumn{8}{|c|}{ 2(a)No. of Capsules/plant at 70 DAS } \\
\hline $\mathrm{T}_{1}$ & Control & 27.07 & 41.08 & 53.37 & 40.5 & & \\
\hline $\mathrm{T}_{2}$ & GA3 @ 100ppm at flowering stage & 31.40 & 44.56 & 54.67 & 43.5 & & \\
\hline $\mathrm{T}_{3}$ & GA3 @ 100ppm at capsule formation stage & 35.67 & 55.78 & 56.90 & 49.4 & & \\
\hline $\mathrm{T}_{4}$ & GA3 @ 100ppm at flowering stage + capsule formation stage & 34.27 & 50.90 & 60.33 & 48.5 & & \\
\hline $\mathrm{T}_{5}$ & IAA @ 100ppm at flowering stage & 37.67 & 57.10 & 71.77 & 55.5 & & \\
\hline $\mathrm{T}_{6}$ & IAA @ 100ppm at capsule formation stage & 36.07 & 56.42 & 70.23 & 54.2 & & \\
\hline $\mathrm{T}_{7}$ & IAA @ 100ppm at flowering stage + capsule formation stage & 33.77 & 51.77 & 80.00 & 55.2 & & \\
\hline $\mathrm{T}_{8}$ & BA@ @ppm at flowering stage & 32.90 & 47.67 & 57.90 & 46.2 & & \\
\hline $\mathrm{T}_{9}$ & BA@ @ppm capsule Formation Stage & 30.07 & 45.33 & 66.90 & 47.4 & & \\
\hline \multirow{4}{*}{$\mathrm{T}_{10}$} & BA @5ppm at flowering stage + capsule formation stage & 33.53 & 54.02 & 63.57 & 50.4 & & \\
\hline & S.Em. \pm & 1.89 & 3.54 & 4.59 & 2.58 & 1.42 & 3.52 \\
\hline & C.D.at $5 \%$ & 5.62 & 10.51 & 13.62 & 7.68 & 4.21 & 9.98 \\
\hline & C.V. \% & 10.86 & 12.14 & 12.49 & 12.41 & & \\
\hline 2(b) & No. of Capsules/plant at Maturity & & & & & & \\
\hline $\mathrm{T}_{1}$ & Control & 30.67 & 53.10 & 57.77 & 47.2 & & \\
\hline $\mathrm{T}_{2}$ & GA3 @ 100ppm at flowering stage & 32.00 & 56.34 & 58.73 & 49.0 & & \\
\hline $\mathrm{T}_{3}$ & GA3 @ 100ppm at capsule formation stage & 37.80 & 68.43 & 59.10 & 55.1 & & \\
\hline $\mathrm{T}_{4}$ & GA3 @ 100ppm at flowering stage + capsule formation stage & 36.93 & 63.57 & 62.00 & 54.2 & & \\
\hline $\mathrm{T}_{5}$ & IAA @ 100ppm at flowering stage & 39.10 & 76.99 & 74.83 & 63.6 & & \\
\hline $\mathrm{T}_{6}$ & IAA@ $100 p p m$ at capsule formation stage & 38.13 & 73.00 & 72.97 & 61.4 & & \\
\hline $\mathrm{T}_{7}$ & IAA @ 100ppm at flowering stage + capsule formation stage & 35.80 & 75.00 & 85.50 & 65.4 & & \\
\hline $\mathrm{T}_{8}$ & BA@ @ppm at flowering stage & 35.70 & 62.89 & 60.17 & 52.9 & & \\
\hline $\mathrm{T}_{9}$ & BA@5ppm capsule Formation Stage & 31.60 & 57.77 & 67.23 & 52.2 & & \\
\hline \multirow[t]{4}{*}{$\mathrm{T}_{10}$} & BA @5ppm at flowering stage + capsule formation stage & 36.77 & 69.67 & 65.30 & 57.2 & & \\
\hline & S.Em. \pm & 1.88 & 4.61 & 4.49 & 2.79 & 1.53 & 3.87 \\
\hline & C.D.at $5 \%$ & 5.59 & 13.71 & 13.34 & 8.30 & 4.55 & 10.99 \\
\hline & C.V. $\%$ & 10.2 & 12.17 & 11.72 & 12.02 & & \\
\hline
\end{tabular}

*Foliar spray at flowering and Capsule formation stage. 
Table.3 Effect of growth regulators on yields of sesame

\begin{tabular}{|c|c|c|c|c|c|c|c|}
\hline Sr. No. & Treatment & 2011 & 2013 & 2014 & Pooled & $\mathbf{Y}$ & $\mathbf{Y} \times \mathbf{T}$ \\
\hline \multicolumn{8}{|c|}{3 (a) Seed Yield $\left(\mathrm{kg} \mathrm{ha}^{-1}\right)$} \\
\hline $\mathrm{T}_{1}$ & Control & 407 & 621 & 1101 & 710 & & \\
\hline $\mathrm{T}_{2}$ & GA3 @ 100ppm at flowering stage & 430 & 614 & 1194 & 746 & & \\
\hline $\mathrm{T}_{3}$ & GA3 @ 100ppm at capsule formation stage & 519 & 794 & 1256 & 856 & & \\
\hline $\mathrm{T}_{4}$ & $\begin{array}{l}\text { GA3 @ 100ppm at flowering stage + } \\
\text { capsule formation stage }\end{array}$ & 461 & 719 & 1313 & 831 & & \\
\hline $\mathrm{T}_{5}$ & IAA@100ppm at flowering stage & 522 & 814 & 1458 & 931 & & \\
\hline $\mathrm{T}_{6}$ & IAA @ 100ppm at capsule formation stage & 495 & 807 & 1414 & 905 & & \\
\hline $\mathrm{T}_{7}$ & $\begin{array}{l}\text { IAA @ 100ppm at flowering stage + } \\
\text { capsule formation stage }\end{array}$ & 557 & 784 & 1502 & 947 & & \\
\hline $\mathrm{T}_{8}$ & BA@5ppm at flowering stage & 452 & 641 & 1267 & 787 & & \\
\hline $\mathrm{T}_{9}$ & BA @ 5ppm capsule Formation Stage & 443 & 638 & 1375 & 819 & & \\
\hline \multirow[t]{4}{*}{$\mathrm{T}_{10}$} & $\begin{array}{l}\text { BA @ } 5 p p m \text { at flowering stage }+ \text { capsule } \\
\text { formation stage }\end{array}$ & 479 & 744 & 1326 & 850 & & \\
\hline & S.Em. \pm & 29 & 49 & 78 & 32 & 17 & 56 \\
\hline & C.D.at $5 \%$ & 88 & 145 & 232 & 94 & 51 & 158 \\
\hline & C.V. $\%$ & 10.7 & 11.8 & 10.2 & 11.5 & & \\
\hline \multicolumn{8}{|c|}{3 (b) Straw Yield $\left(\mathrm{kg} \mathrm{ha}^{-1}\right)$} \\
\hline $\mathrm{T}_{1}$ & Control & 1698 & 2148 & 2438 & 2095 & & \\
\hline $\mathrm{T}_{2}$ & GA3 @ 100ppm at flowering stage & 1731 & 2130 & 2469 & 2110 & & \\
\hline $\mathrm{T}_{3}$ & GA3 @ 100ppm at capsule formation stage & 2096 & 2623 & 2531 & 2417 & & \\
\hline $\mathrm{T}_{4}$ & $\begin{array}{l}\text { GA3 @ 100ppm at flowering stage + } \\
\text { capsule formation stage }\end{array}$ & 2052 & 2593 & 2809 & 2485 & & \\
\hline $\mathrm{T}_{5}$ & IAA@100ppm at flowering stage & 2299 & 2778 & 3179 & 2752 & & \\
\hline $\mathrm{T}_{6}$ & IAA @ 100ppm at capsule formation stage & 2123 & 2654 & 3086 & 2621 & & \\
\hline $\mathrm{T}_{7}$ & $\begin{array}{l}\text { IAA @ 100ppm at flowering stage + } \\
\text { capsule formation stage }\end{array}$ & 2333 & 2840 & 3256 & 2810 & & \\
\hline $\mathrm{T}_{8}$ & BA@5ppm at flowering stage & 1796 & 2099 & 2623 & 2173 & & \\
\hline $\mathrm{T}_{9}$ & BA@ @ppm capsule Formation Stage & 1914 & 2284 & 2901 & 2366 & & \\
\hline \multirow[t]{4}{*}{$\mathrm{T}_{10}$} & $\begin{array}{l}\text { BA @ } @ \text { ppm at flowering stage }+ \text { capsule } \\
\text { formation stage }\end{array}$ & 1963 & 2377 & 2716 & 2352 & & \\
\hline & S.Em. \pm & 130 & 178 & 189 & 62 & 34 & 167 \\
\hline & C.D.at $5 \%$ & 385 & 527 & 560 & 184 & 101 & 474 \\
\hline & C.V. \% & 11.2 & 12.5 & 11.7 & 12.0 & & \\
\hline
\end{tabular}

*Foliar spray at flowering and Capsule formation stage. 
Table.4 Effect of growth regulators on harvest Index

\begin{tabular}{|c|c|c|c|c|c|c|c|}
\hline Sr. No & Treatment & 2011 & 2013 & 2014 & Pooled & $\mathbf{Y}$ & $\mathbf{Y} \mathbf{x} \mathbf{T}$ \\
\hline $\mathrm{T}_{1}$ & Control & 19.4 & 22.7 & 31.2 & 24.4 & & \\
\hline $\mathrm{T}_{2}$ & GA3 @ 100ppm at flowering stage & 19.9 & 22.4 & 32.5 & 24.9 & & \\
\hline $\mathrm{T}_{3}$ & GA3 @ 100ppm at capsule formation stage & 20.7 & 23.1 & 33.4 & 25.8 & & \\
\hline $\mathrm{T}_{4}$ & GA3 @ 100ppm at flowering stage + capsule & 185 & 218 & 320 & 241 & & \\
\hline $\mathrm{T}_{5}$ & IAA @ 100ppm at flowering stage & 18.8 & 22.7 & 31.6 & 24.4 & & \\
\hline $\mathrm{T}_{6}$ & IAA@100ppm at capsule formation stage & 19.0 & 23.3 & 31.4 & 24.6 & & \\
\hline $\mathrm{T}_{7}$ & $\begin{array}{l}\text { IAA @ } 100 \mathrm{ppm} \text { at flowering stage + capsule } \\
\text { formation stage }\end{array}$ & 19.4 & 21.8 & 31.6 & 24.2 & & \\
\hline $\mathrm{T}_{8}$ & BA@ @ppm at flowering stage & 20.4 & 23.4 & 32.9 & 25.5 & & \\
\hline $\mathrm{T}_{9}$ & BA @ 5ppm capsule Formation Stage & 19.0 & 21.7 & 32.6 & 24.4 & & \\
\hline $\mathrm{T}_{10}$ & $\begin{array}{l}\text { BA @5ppm at flowering stage }+ \text { capsule } \\
\text { formation stage }\end{array}$ & 20.0 & 24.1 & 33.0 & 25.7 & & \\
\hline & S.Em. \pm & 1.3 & 1.6 & 2.1 & 0.3 & 0.17 & 1.73 \\
\hline & C.D.at $5 \%$ & NS & NS & NS & 0.9 & 0.49 & 4.91 \\
\hline & C.V. $\%$ & 11.8 & 12.4 & 11.5 & 12.1 & & \\
\hline
\end{tabular}

*Foliar spray at flowering and Capsule formation stage

Table.5 Effect of growth regulators on 1000 seed weight (g) of sesame

\begin{tabular}{|c|c|c|c|c|c|c|c|}
\hline Sr. No & Treatment & 2011 & 2013 & 2014 & Pooled & $\mathbf{Y}$ & $\mathbf{Y} \mathbf{x}$ \\
\hline $\mathrm{T}_{1}$ & Control & 2.38 & 3.07 & 3.17 & 2.87 & & \\
\hline $\mathrm{T}_{2}$ & GA3 @ 100ppm at flowering stage & 2.71 & 3.15 & 3.20 & 3.02 & & \\
\hline $\mathrm{T}_{3}$ & GA3 @ 100ppm at capsule formation stage & 2.78 & 3.31 & 3.22 & 3.10 & & \\
\hline $\mathrm{T}_{4}$ & $\begin{array}{l}\text { GA3 @ 100ppm at flowering stage + capsule } \\
\text { formation stage }\end{array}$ & 2.71 & 3.19 & 3.27 & 3.06 & & \\
\hline $\mathrm{T}_{5}$ & IAA @ 100ppm at flowering stage & 2.84 & 3.35 & 3.47 & 3.22 & & \\
\hline $\mathrm{T}_{6}$ & IAA @ 100ppm at capsule formation stage & 2.91 & 3.30 & 3.43 & 3.21 & & \\
\hline $\mathrm{T}_{7}$ & $\begin{array}{l}\text { IAA @ 100ppm at flowering stage + capsule } \\
\text { formation stage }\end{array}$ & 3.12 & 3.51 & 3.50 & 3.38 & & \\
\hline $\mathrm{T}_{8}$ & BA@ @ ppm at flowering stage & 2.63 & 3.17 & 3.25 & 3.02 & & \\
\hline $\mathrm{T}_{9}$ & BA @ 5ppm capsule Formation Stage & 2.64 & 3.16 & 3.38 & 3.06 & & \\
\hline $\mathrm{T}_{10}$ & $\begin{array}{l}\text { BA @ @ 5pm at flowering stage }+ \text { capsule formation } \\
\text { stage }\end{array}$ & 2.71 & 3.28 & 3.32 & 3.10 & & \\
\hline & S.Em. \pm & 0.06 & 0.07 & 0.07 & 0.04 & 0.02 & 0.07 \\
\hline & C.D.at $5 \%$ & 0.19 & 0.22 & 0.22 & 0.12 & 0.07 & 0.20 \\
\hline & C.V.\% & 3.99 & 3.93 & 3.82 & 3.92 & & \\
\hline
\end{tabular}

*Foliar spray at flowering and Capsule formation stage.

\section{Effect of growth regulators on yield}

Effect of growth regulators on yields of sesame is presented in Table 3.The yields of sesame were significantly differed during all the three year and also in pooled result due to foliar spraying of growth regulator. On the bases of pooled results, maximum seed yield and straw yield were $947 \mathrm{~kg}$ $\mathrm{ha}^{-1}$ (Table 3a) and $2810 \mathrm{~kg} \mathrm{ha}^{-1}$ (Table 3b) respectively obtained due to foliar spraying of
IAA @ 100 ppm at flowering and capsule formation stage (T7) and statically at par T5 and T6. Results (Table 3) further indicated that around $34 \%$ increase in seeds, stalk and biological yield were recorded with $\mathrm{T} 7$ in comparison to their respective control. The lowest seed yield $(710 \mathrm{~kg}$ $\left.\mathrm{ha}^{-1}\right)$ and straw yield $\left(2095 \mathrm{~kg} \mathrm{ha}^{-1}\right)$ were recorded under control treatment. Rastogi et al., (2013) effort done in linseed (Linumusitatis simum L.) and get better result with indole acetic acid. 


\section{Effect of growth regulators on harvest index and 1000 seed weight $(\mathrm{g})$}

The effect of different treatments on harvest index was found significant and varied from 24.1 to 25.8 (Table 4). The significantly highest harvest index was recorded under treatment $\mathrm{T}_{3}\left(\mathrm{GA}_{3} @ 100 \mathrm{ppm}\right.$ at capsule formation stage) followed by $\mathrm{T}_{2}$. The significantly highest1000 seed weight (3.38 g) of sesame was recorded due to spraying of IAA @ 100 ppm at flowering and capsules formation stage(T7,Table 5). The least harvest index was notedunder control treatment. Maximum result was found in IAA at $100 \mathrm{ppm}$ at flowering and capsules formation may be due to its plays an important role in improving the plant growth, development and yield of crops and affects the physiological processes, hastens maturity and improving the quality of fruits (Rout, 2006; Khaled et al., 2015). Growth hormone like Indole acetic acid is naturally occurring auxin that stimulates growth through cell elongation and lateral root formation which probably support more absorption of minerals (Egamberdieva, 2009; Rathod et al., 2015).

From the overall results it may be concluded that IAA treated plot at flowering stage showed better performance for growth parameters and yield compared to control condition. Therefore, $100 \mathrm{ppm}$ IAA at flowering stage was more suitable than the other treatments for sesame cultivation.

\section{References}

Egamberdieva, D. 2009. Alleviation of salt stress by plant growth regulators and IAA producing bacteria in wheat, Acta Physiologiae Plantarum, 31: 861-864.

Garai, A.K., and Datta, K. 1999. Influence of plant growth regulators on growth, morphophysiological characters and yield of summer sesame (Sesamum indicum L. cv. Rama) under moisture stress. Acta Physiologiae Plantarum, 21: 277-281.

Joshi, G., Shukla, A., and Shukla, A. 2011. Synergistic response of auxin and ethylene on physiology of Jatropha curcas L. Brazilian J.
Plant Physiol., 23: 1-7.

Khaled, M., Sikder, S., Islam, M.R., Hasan, M.A., and Bahadur, M.M. 2015. Growth yield and yield attributes of tomato (Lycopersiconesculentum Mill.) as influenced by indole acetic acid. J. Environ. Sci. Natural Res., 8(1): 139-145.

Lee, H.S. 1990. Effects of presowing seed treatments with GA3 and IAA on flowering and yield components in groundnuts. Korean J. Crop Sci., 35: 19.

Quaderi, R.S., Islam, S.A., Faruque, A.F.M.G., Hoque, M., and Haque, S. 2006. Influence of seed treatment with indole acetic acid on mungbean cultivation. Int. J. Bot., 2: 4247.

Rahman, M.M., Islam, M.A. and Mondal, M. R.K. 1989. Effect of wave length of light and some phytohormones on the growth and yield of grass pea. Bangladesh J. Agri. Res., 14: 1923.

Rastogi, A., Siddiqui, A., Mishra, B. K., Srivastava, M., Pandey, R., Misra, P., Singh, M., and Shukla, S. 2013. Effect of auxin and gibberellic acid on growth andyield components of linseed (Linumusitatissimum L. Crop Breeding Appl. Biotechnol., 13: 1-7.

Rathod, R.R., Gore, R.V., and Bothikar, P.A. 2015. Effect of Growth Regulators on Growth and Yield of French bean (Phaseolus vulgaris L.) Var. Arka Komal. IOSR J. Agri. Vet. Sci., 8: 36-39.

Reena, T., Delotale, R.D., Armarkar, N., and Chore, C.N. 1999. Influence of seed soaking in IAA and kinetin solutions of growth and yield of soybean. J. Soils Crops, 9: 7277.

Rout, G.R. 2006. Effects of Auxins on adventitious root development from single node cutting of Camellia sinensis (L.) Kuntze and associated biochemical changes. Plant Growth Regulators, 48: 111-117.

Senthil, A., Pathmanaban, G., Srinivasan, P.S. 2003. Effect bioregulators on some physiological and biochemical parameters of soybean (Glycine max L. Legume Res. India, 26: 5456.

\section{How to cite this article:}

Vekaria, G.B., K.D. Rakholiya, V.D. Vora, J.T. Patel, G.S. Sutaria and Vekaria, P.D. 2017. Response of Sesame (Sesamum indicum L.) to Growth Regulator under Dry Farming Condition. Int.J.Curr.Microbiol.App.Sci. 6(3): 1113-1120. doi: https://doi.org/10.20546/ijcmas.2017.603.129 